\title{
EVALUACIÓN DEL COMPORTAMIENTO HIGIÉNICO DE APIS MELLIFERA L. EN RELACIÓN AL NIVEL DE INFESTACIÓN DE VARROA DESTRUCTOR ANDERSON \& TRUEMAN
}

\author{
EVALUATION OF THE HYGIENIC BEHAVIOR OF APIS MELLIFERA \\ L. RELATED TO THE LEVEL OF INFESTATION OF VARROA DESTRUCTOR \\ ANDERSON \& TRUEMAN
}

Ximena Araneda D. ${ }^{1}$; Roberto Pérez $N .{ }^{1}$; Claudia Castillo R. $^{1}$; Leovigildo Medina M. ${ }^{1}$

\section{RESUMEN}

\begin{abstract}
La apicultura en Chile ha sido importante sobre todo para la pequeña y mediana agricultura, por los diversos productos que se pueden extraer de esta explotación, pero uno de los mayores problemas son la incidencia de enfermedades y parásitos. Una de las principales patologías es el ácaro ectoparásito Varroa destructor Anderson \& Trueman, debido a su difícil control, ya que se reproduce en celdas operculadas de cría, disminuyendo la población de la colonia y en infestaciones severas haciéndola desaparecer por completo; además de la utilización de productos químicos para su control, los cuales no son usados correctamente, creando resistencia y dejando residuos en los productos de origen apícola. Para revertir esta situación se hace necesario buscar nuevas alternativas de control, tales como la resistencia genética, por medio del comportamiento higiénico.

En este estudio se analiza el comportamiento higiénico en relación al nivel de infestación de Varroa destructor. Para la elaboración de este estudio se utilizaron veintiuna colonias de abejas melíferas, las cuales se evaluaron desde octubre a diciembre del año 2005. A cada colonia se le determinó el porcentaje de comportamiento higiénico a través de la técnica del perforado; conjuntamente se tomaron muestras para realizar los diagnósticos de detección de Varroa.

Los resultados evidenciaron un bajo y heterogéneo nivel de comportamiento higiénico, donde los valores fluctuaron en promedio en las colonias desde $20 \%$ a $80 \%$ ( 2 colonias) y la inexistencia de correlación.

Los parámetros evaluados presentaron infestaciones promedio de: abeja adulta 4,5\%; cría obrera 7\% y cría de zángano $31 \%$.

Palabras claves: Comportamiento higiénico, Varroa destructor, Apis mellifera.
\end{abstract}

\begin{abstract}
The beekeeping in Chile has been especially important for the small and medium agriculture due to the several products that can be obtained from the explotation. One of the major problems is the incidence of diseases and parasites, being the acarus ectoparasite Varroa destructor Anderson \& Trueman one of the main pathologies because of its difficult control since it's reproduced in brood capped cells, decreasing the population of the colony and in strong infestations making them disappear completely; also by using chemical products for its control, which are not used correctly, producing resistance and leaving residues in the bee products. In order to change this situation it is necessary to find new control alternatives such as genetic resistance through hygienic behavior. The hygienic behavior related to the infestation level of Varroa destructor is analyzed in this work.

Twenty one honeybee colonies were involved in this study, which were evaluated from October to December 2005. The percentage of hygienic behavior of each colony was determined through the pin-killed test. Samples to carry out the diagnoses of varroa were also taken.

The results showed a low and heterogeneous level of hygienic behavior, where the values fluctuated in the colonies in average from $20 \%$ to $80 \%$ (2 colonies). The non-existence of correlation was also shown.

The evaluated parameters showed an infestation average of $4.5 \%$ in adult bee; $7 \%$ in worker brood and $31 \%$ in drone brood.
\end{abstract}

Key words: Hygienic behavior, Varroa destructor, Apis mellifera.

$1 \quad$ Universidad Católica de Temuco, Temuco, Chile. E-mail: xaraneda@uct.cl

Fecha de Recepción: 27 Diciembre 2006

Fecha de Aceptación: 06 Mayo 2007 


\section{INTRODUCCIÓN}

La apicultura en Chile ha sido importante, sobre todo para la pequeña y mediana agricultura, a través de los años, por la variada oferta de productos relacionados con esta actividad, como son los extraídos directamente de la colmena (miel, polen, propóleo, jalea real), además de su utilización en cultivos industriales como agentes polinizadores y recientemente la extracción de apitoxinas para fines terapéuticos.

En un comienzo la apicultura mayoritariamente se desarrollaba en colmenas rústicas, pero con el paso del tiempo la incidencia de enfermedades hizo desaparecer esta forma de explotación; el principal causante de este cambio fue la llegada del ácaro ectoparásito llamado primeramente Varroa jacobsoni Oud. y, actualmente, Varroa destructor Anderson \& Trueman.

Varroa destructor parasita dos especies de abejas: Apis cerana y Apis mellifera. Cabe destacar que sobre Apis cerana el ácaro no causa daños graves, fundamentalmente debido a que sólo se reproduce en celdas de cría de zángano. Por el contrario, la interacción entre Varroa y Apis mellifera no se encuentra en equilibrio, ya que en esta especie el ácaro tiene la capacidad de reproducirse tanto en celdas de zángano como de obreras. Por esta razón la reproducción es mucho mayor y, por lo tanto, puede llegar a causar la muerte de las colonias.

Según Vandame (2000), afecta a la abeja melífera en todos sus estadios de desarrollo (cría abierta, sellada e individuo adulto). Kralj (2004) al respecto menciona que el daño causado a la cría está relacionado con la muerte de abejas, nacimiento de abejas con alas atrofiadas, menores pesos y reducción en la vida de las obreras.

Además de la infestación en sí, una de las mayores problemáticas en la apicultura de nuestro país en la actualidad es la aplicación de productos químicos para su control, en dosis no adecuadas $\mathrm{y}$ de productos no recomendados para el uso en abejas.

Según Jong et al. (1984), Robaux (1988), Vicario y Medina (1999) la utilización de productos químicos presenta una serie de inconvenientes que limitan su uso en las colonias, ya que la mayoría de estos contaminan la miel y la cera de las colonias medicadas, ocasionan intoxicaciones a las abejas y al apicultor, presentan un alto costo por tratamiento y después de algunos años de exposición al producto se observa la aparición de ácaros resistentes.

Existen dos conductas de la abeja Apis mellífera asociadas a la resistencia a enfermedades, una es el comportamiento de grooming o acicalamiento que se define según Flores et al. (1998) como la capacidad de las abejas de detectar, morder y eliminar los parásitos en etapa forética y es una posible vía para la selección de abejas tolerantes a enfermedades, aunque no la única. Este comportamiento de aseo puede ser sobre sí misma y se conoce como autogrooming, donde la abeja cepilla su cuerpo entero con sus extremidades; también este comportamiento puede ser sobre otras abejas, denominándose allogrooming, en el cual una o más abejas buscan el ácaro en la abeja infestada, tomándolo con sus mandíbulas y arrojándolo al piso de la colmena.

La otra conducta asociada con la resistencia a enfermedades es el comportamiento higiénico. Andere et al. (2001) lo definen como la habilidad de las obreras de desopercular las celdas y remover la cría muerta de su interior y ha sido relacionado con la resistencia a las enfermedades ya que permite a las abejas eliminar la masa infectante de la colonia. Al respecto Palacio et al. (2000), Gramacho y Gonçalves (2002) señalan además que una colonia para ser considerada como higiénica debe presentar un valor de remoción de la cría mayor o igual a $80 \%$. Otros autores como Spivak y Reuter (2001), Kamel et al. (2003), Ibrahim y Spivak (2006) consideran higiénicas a aquellas abejas que remueven sobre el 95\% de las crías, siendo este un rasgo deseable, puesto que las abejas higiénicas rápidamente interrumpen el ciclo contagioso.

El comportamiento higiénico es un mecanismo de resistencia para diferentes patologías tales como: loque americano (Spivak y Reuter 1997), cría de tiza (Gilliam et al. 1983) y Varroa destructor (Boecking y Drescher, 1992; Palacio et al., 2005).

Según Andere et al. (2001), la selección por este comportamiento permite obtener abejas tolerantes a enfermedades, y de esta manera disminuir la propagación e incidencia de las mismas en las colonias evitando el uso de químicos.

Arechavaleta y Guzmán-Novoa (2001) señalan que disminuyendo el uso de químicos se reducen los costos de producción de miel.

Para la determinación de este comportamiento los métodos más utilizados son: el congelado y el perforado (Palacio et al., 1996; Invernizzi, 2001; 
Kralj, 2004). Gramacho y Gonçalves (1994) compararon estos dos métodos, concluyendo que el método del perforado es más eficaz en términos de cantidad de crías muertas removidas. Recomendando entonces la utilización de éste, por ser más rápido, eficaz, práctico y económico que el congelado, además, pudiendo ser fácilmente aplicado a nivel de campo.

Considerando los problemas ocasionados por la presencia de Varroa en las colonias, se hace necesario estudiar y analizar la relación existente entre el comportamiento higiénico y la resistencia a este parásito.

En este estudio el objetivo principal es establecer la existencia de relación entre comportamiento higiénico de Apis mellifera e infestación de varroa y, como objetivos secundarios, evaluar este comportamiento en abeja adulta, celdas de obreras y zánganos, para relacionarlo con la variación de la infestación del ácaro.

\section{MATERIALES Y MÉTODOS}

Se eligieron 21 colonias de abejas europeas de la especie Apis mellifera L., en colmenas tipo Langstroth.

A cada colonia se le determinó el comportamiento higiénico a través de la técnica del perforado de las crías descrito por Newton y Ostasiewski (1986), modificado por Gramacho y Gonçalves (1994).

Se utilizó la metodología descrita por Vandame (2000) para determinar infestación de varroa en abeja adulta y cría de obrera y zángano.

Para la toma de muestras se realizaron 4 repeticiones con un mínimo de 15 días de separación entre ellas.

Al mismo tiempo que se realizó la técnica para determinar comportamiento higiénico, se tomaron muestras para realizar los diagnósticos de detección de varroa. Esto último con el fin de ver la relación que existe entre el comportamiento higiénico y la infestación de varroa.

Para el análisis estadístico se utilizó el programa SPSS (Stadistical Product and Service Solutions) para Windows en su versión 12.0.

Los resultados obtenidos de cada parámetro evaluado en la investigación fueron analizados a través de un análisis de varianza (ANDEVA) de un factor $(\mathrm{p}<0,05)$, pruebas no paramétricas para $\mathrm{K}$ muestras independientes ( $\mathrm{H}$ de Kruskal-Wallis), pruebas de correlación no paramétricas (Rho de Spearman) y pruebas de comparación múltiples de promedios (Tuckey HSD).

\section{RESULTADOS Y DISCUSIÓN}

Los resultados obtenidos del análisis estadístico de correlación para variables no paramétricas de Spearman entre comportamiento higiénico con los grados de infestación de varroa en abejas adultas, infestación de cría de obrera e infestación de cría de zángano dio como resultado que no hay correlación.

Los resultados obtenidos en este estudio concuerdan con lo realizado por Mondragón et al. (2005), donde no encontraron ninguna relación significativa en la fluctuación del número de ácaros y el comportamiento higiénico, a lo largo de un período de tres meses del año de evaluación.

Arechavaleta y Guzmán-Novoa (2001) al evaluar niveles de infestación de varroa y el número de celdas limpiadas por abejas, no encontraron relación entre estas variables. Estos autores hacen referencia a que existiría una correlación negativa entre el grado de comportamiento higiénico y la susceptibilidad del parásito Varroa destructor Anderson \& Trueman.

En contraste, Moretto (1996) encontró una alta correlación $(\mathrm{r}=-0,7)$ respecto a la influencia del comportamiento higiénico y la variación en la población de varroa, en abejas africanizadas; dejando de manifiesto la mayor resistencia hacia el parásito.

Gonçalves y Gramacho (2000) al respecto agregan que las abejas africanizadas son siete veces más eficientes en la remoción de ácaros de sus colonias que las abejas italianas. Por otro lado, Vieira y Gonçalves (2000) señalan que abejas africanizadas en México remueven $32 \%$ de las crías infestadas naturalmente por varroas, comparado con un $8 \%$ de remoción en colonias de abejas europeas.

Lo anteriormente expuesto coincidiría con los resultados obtenidos, los cuales evidencian una relación negativa entre el comportamiento higiénico y el nivel de infestación del parásito, pudiéndose pensar que el poseer abejas con mayor capacidad higiénica la relación puede ser más significativa.

Los resultados obtenidos en el estudio pueden atribuirse al bajo y heterogéneo nivel de comportamiento higiénico evidenciado por las colonias, 
fuentes de reinfestación que pueden ser por intermedio de zánganos, los cuales actuarían según Benítez y Medina (2001) entrando libremente a las colmenas, así como también por la deriva de abejas cuando están iniciando su actividad de pecoreo, además de las abejas que roban cuando hay escasez de alimento o colonias débiles (pillaje del mismo apiario o de apiarios circundantes) y enjambres silvestres cercanos al apiario del apicultor.

Al respecto, Chávez y García (1993) señalan que las principales causas de expansión de varroa son: el pillaje, la deriva, el ir y venir de los zánganos, las manipulaciones descuidadas del apicultor y la trashumancia no controlada de las colmenas, a lo que hay que añadir la falta de detección precoz de la enfermedad.

Vandame et al. (2000) afirman que en un día de alta actividad de las pecoreadoras, hasta 70 varroas pueden llegar a una nueva colmena.

Al comparar el comportamiento higiénico entre colonias, se obtuvo como resultado que no hay diferencias estadísticamente significativas $(\mathrm{p}<0,005)$.

La Figura 1 muestra lo bajo de este comportamiento, ya que sólo 2 colonias superaron en promedio el 80\%; además, se observa una alta heterogeneidad, ya que en la mayoría de las colonias se obtuvieron diferencias entre las repeticiones, lo que puede deberse a que la reina usualmente copula con diez a diecisiete zánganos, para poder llenar su espermateca, donde guarda estos espermas en forma de paquetes los que va usando en forma alternada, los cuales pueden no poseer la característica higiénica (Palacio et al., 2000).

Andere et al. (2001) señalan que las variables climáticas podrían ejercer una influencia sobre el comportamiento higiénico.

Hueraman (2002) hace referencia a que no existen diferencias en el comportamiento higiénico en un estudio realizado comparando días soleados, nublados y lluviosos, recomendando realizar evaluaciones de comportamiento higiénico en días soleados, evitando los días nublados y lluviosos, puesto que se dificulta el manejo apícola.

Otro factor es la incidencia del flujo nectario, el cual enmascararía comportamientos higiénicos bajos, es decir, que al aumentar el flujo nectario aumentaría la limpieza en la colmena pero no por eliminar enfermedades sino para prepararla para la recepción de néctar.

Janmaat y Winston (2000) agregan que al existir una alta oferta de néctar y por una actitud de atesoramiento de reservas por la colmena, las obreras optan por remover nidada, lo cual evita el

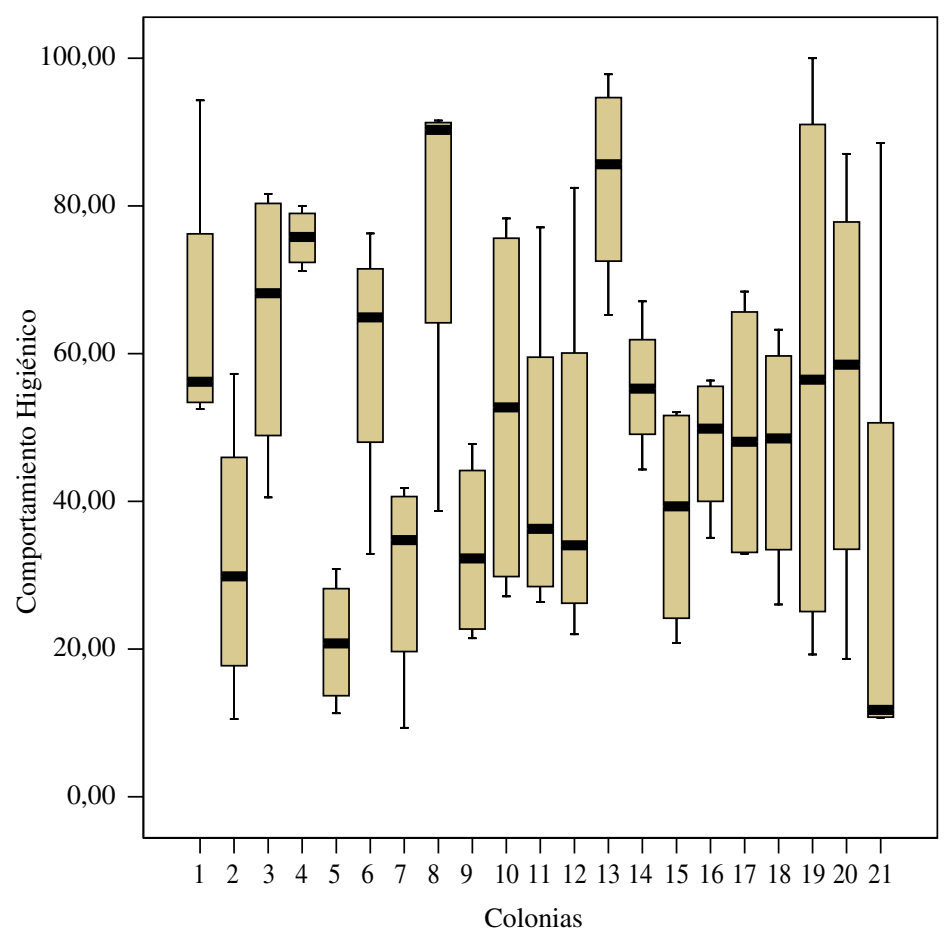

Figura 1. Variaciones del comportamiento higiénico ocurridas en cada colonia. 
esfuerzo de construcción de nuevas celdas y, por consiguiente, esta actividad incrementa el comportamiento higiénico.

Otro factor que pudo tener influir en lo bajo $\mathrm{y}$ variable del comportamiento es la procedencia de las colonias, donde la mayoría fue obtenida de enjambres o no fueron seleccionadas para el comportamiento higiénico.

Además, se debe considerar la baja frecuencia de este comportamiento, es decir, se presenta aproximadamente en un $10 \%$ de las colonias de Apis mellifera en poblaciones naturales (Spivak y Gilliam, 1993; Oldroyd, 1996; Spivak y Downey, 1998).

La Figura 2 muestra que al igual que el parámetro anteriormente evaluado, no existen diferencias estadísticamente significativas entre colonias, ya que se puede observar que el rango en que se desplazan es de 3 a 7\%; a su vez muestra una baja heterogeneidad de la tasa de infestación en adultos presentes en las colonias, donde la mayoría tuvo una menor diferencia entre las repeticiones, lo que evidencia que la presencia de varroa en etapa forética se mantuvo baja y no varió en el tiempo de evaluación. Esto podría deberse al control del ácaro que se realizó en primavera, el cual puede tener efecto en la baja variación de la población. Además, al ser un apiario experimental no se recurre a la nivelación de colonias, por medio del intercambio de marcos con cría.

Otro factor que podría incidir es el comportamiento de grooming (el cual no fue evaluado en este estudio). Este es un mecanismo de limpieza utilizado por las abejas cuando las varroas están en etapa forética. La abeja es capaz de detectar los parásitos introducidos en celdillas de obreras, retirarlos y eliminarlos; también localiza los parásitos sobre las abejas adultas, los muerde y elimina (Flores et al. 1998).

Según Vandame et al. (2000) las hembras de Varroa en etapa forética tienen una preferencia por las abejas nodrizas, las que por su labor en la colmena tienen mayor cercanía a la cría, lo que ofrece más oportunidades a los ácaros para entrar en la celda cría.

Respecto al parámetro de infestación de varroa en cría de obrera al igual que en los anteriores no existen diferencias estadísticamente significativas entre colonias; además, se puede observar que los promedios de infestación de varroas en crías de obreras intracolonia presentan en la mayoría homogeneidad entre repeticiones, aunque en las colonias 4,11 y 15 estos rangos son más amplios (Figura 3).

Una explicación de este fenómeno sería la variación en la cantidad de cría de obrera, puesto que una disminución de ésta da como resultado un incremento en las celdas que contienen varroa; por un efecto de dilución, consecuentemente al haber menos crías la infestación sería mayor (Medina et al. 2002).

El volumen de cría va a depender de la cantidad de huevos que la reina pueda poner al día. Esta cantidad se relaciona con circunstancias tales como: la estación y recolección, donde la postura es proporcional al trabajo de recolección que desarrollan las abejas. Así en invierno la postura es casi nula; también depende de la edad de las reinas, ya que ponen la mayoría de los huevos en los dos primeros años de vida. Además depende del espacio disponible para los huevos y también de la cantidad de obreras de la colonia, ya que deben haber suficientes abejas para cuidar todas las crías (De Layens y Bonnier 2001).

Duay et al. (2003) hacen referencia que en cría de obrera, 4 a 6 varroas hembras, en la mayoría de los casos impiden el nacimiento de la abeja, lo cual mermaría la cantidad de éstas.

$\mathrm{Al}$ evaluar los promedios de infestación de varroas en crías de obreras intercolonias, se observa nuevamente que los rangos se mantienen entre 3 y $10 \%$. Esto puede deberse a que la varroa, como cualquier otro parásito, mantiene los umbrales estables de infestación no causando daños severos, prolongando la vida del huésped.

Estos niveles de infestación, según Vandame (2000), están al límite de requerir un control inmediato.

Estudios realizados por Vandame et al. (2000) revelan que dos terceras partes de las varroa entran en la cría de abejas europeas, permaneciendo una tercera parte en etapa forética; en contraste, dos terceras partes de las abejas africanizadas permanecieron foréticas, mostrando estas últimas mayor tolerancia al parásito.

Otro factor que puede tener efecto en la variación de la población de varroa en celdas de cría de abejas es la supresión de la reproducción del ácaro (SMR). Harbo y Harris (1999) lo definen como un carácter genético que poseen las abejas que causa que los ácaros se vuelvan no reproductivos. Esto quiere decir que la hembra que entra en la celda produce sólo crías estériles o machos. Además señalan que por 


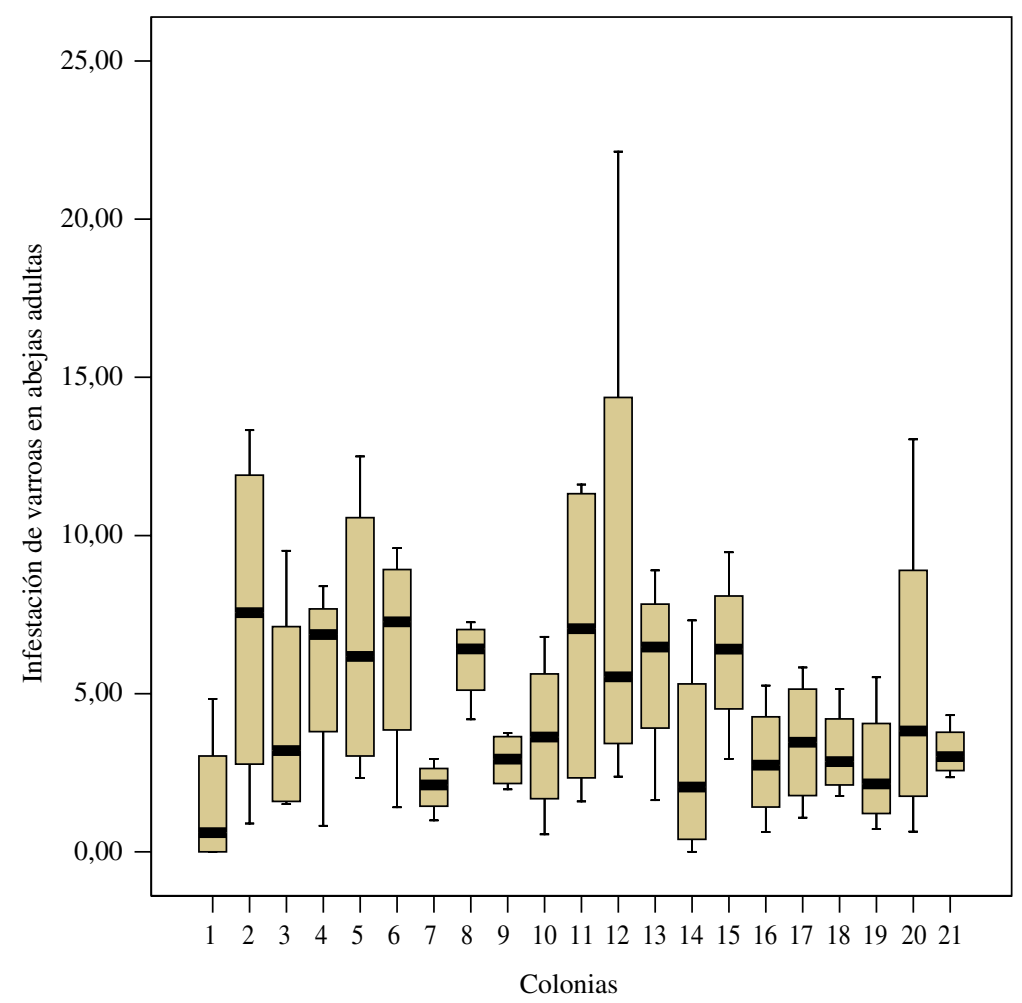

Figura 2. Porcentaje de infestación de varroas en abejas adultas por colonia.

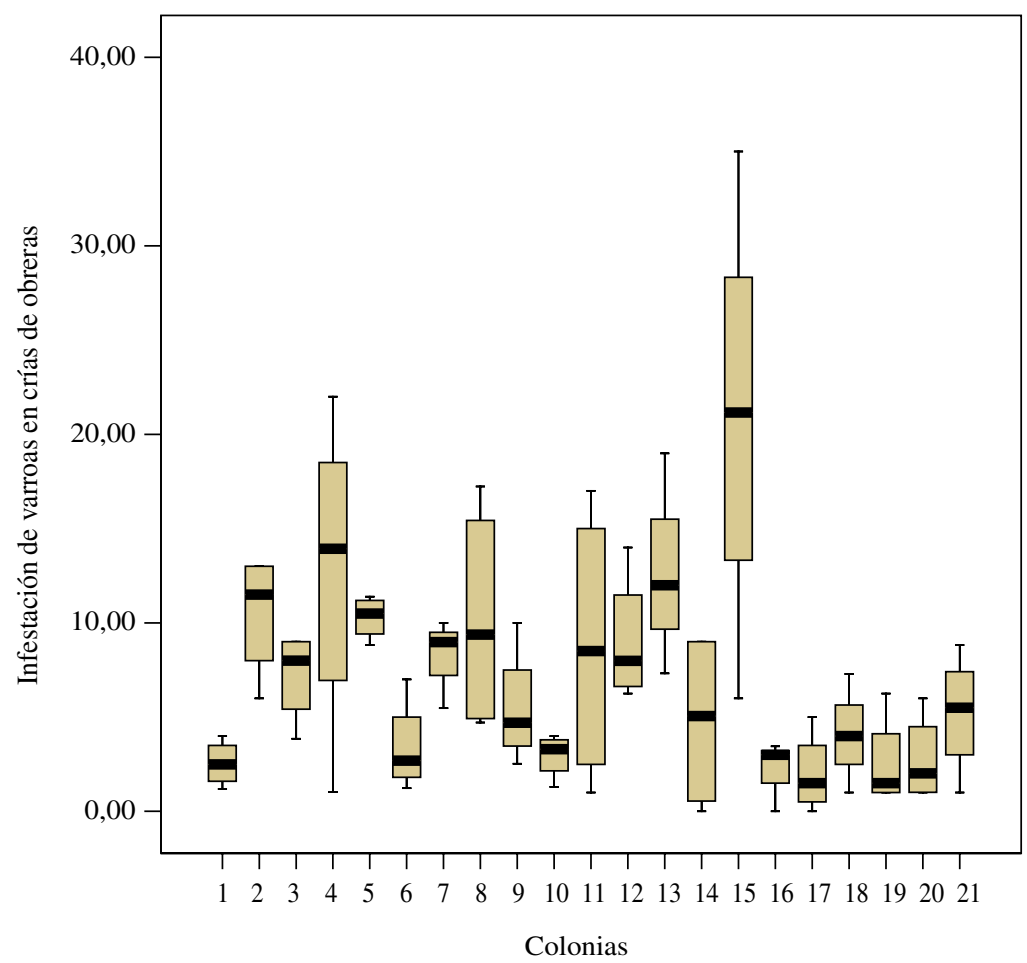

Figura 3. Porcentaje de infestación de varroas en crías de obreras por colonia. 
medio de selección han aumentado la expresión de este carácter de $40 \%$ (frecuencia máxima de ácaros no reproductivos en abejas europeas) al 100\%.

Respecto al parámetro de infestación de varroas en crías de zánganos, al igual que en los anteriores no existen diferencias estadísticamente significativas entre colonias. Al observar la Figura 4 queda de manifiesto que los valores de infestación son muy superiores a los vistos en cría de obrera, encontrándose la mayoría de las colonias sobre el $20 \%$.

La fecundación de las hembras de varroa se desarrolla en el interior de la celda operculada. Para ello eligen una que contenga una larva finalizando su etapa abierta, teniendo preferencia por las de zánganos. Esta preferencia según Chávez y García (1993) es debida a que el mayor tamaño de las celdillas de zánganos le proporciona más alimento, por lo que se deposita un mayor número de huevos. La menor temperatura de estas celdas, por estar en los bordes del panal, facilita la multiplicación del parásito, siendo los zánganos los más afectados llegando a desaparecer su capacidad de fecundación.

Calderone et al. (2002) agregan que el mayor tiempo de duración de la etapa larval que presentan los zánganos respecto a las obreras tendría influen- cia en la predilección de las varroas por las crías de zánganos.

Duay et al. (2003) hacen referencia que algunos zánganos son capaces de emerger aun con cerca de 20 varroas fértiles, lo cual aumentaría la infestación de la colonia.

Referente a la presencia de cría de zángano, esta fue muy variable en cantidad y en algunos casos estuvo ausente, lo cual concuerda con el estudio realizado por Mondragón et al. (2005).

Además, los resultados obtenidos muestran que la infestación de cría de zángano en promedio es 4 veces más alta que la de obrera.

Los niveles elevados de varroa en cría de zángano hacen necesario al momento de hacer evaluaciones de infestación, no sólo muestrear cría de obrera, sino también de zánganos en los momentos de mayor presencia en la colmena.

Por lo antes mencionado, para poder disminuir la intensidad de los ataques de varroa, la resistencia genética de las abejas es una buena alternativa de selección. Pero hace falta hacer estudios multifactoriales donde se evalúen paralelamente los efectos del comportamiento de grooming, comportamiento higiénico, supresión de la reproducción del ácaro (SMR), para así ver el efecto real que poseen éstos en la población de varroa.

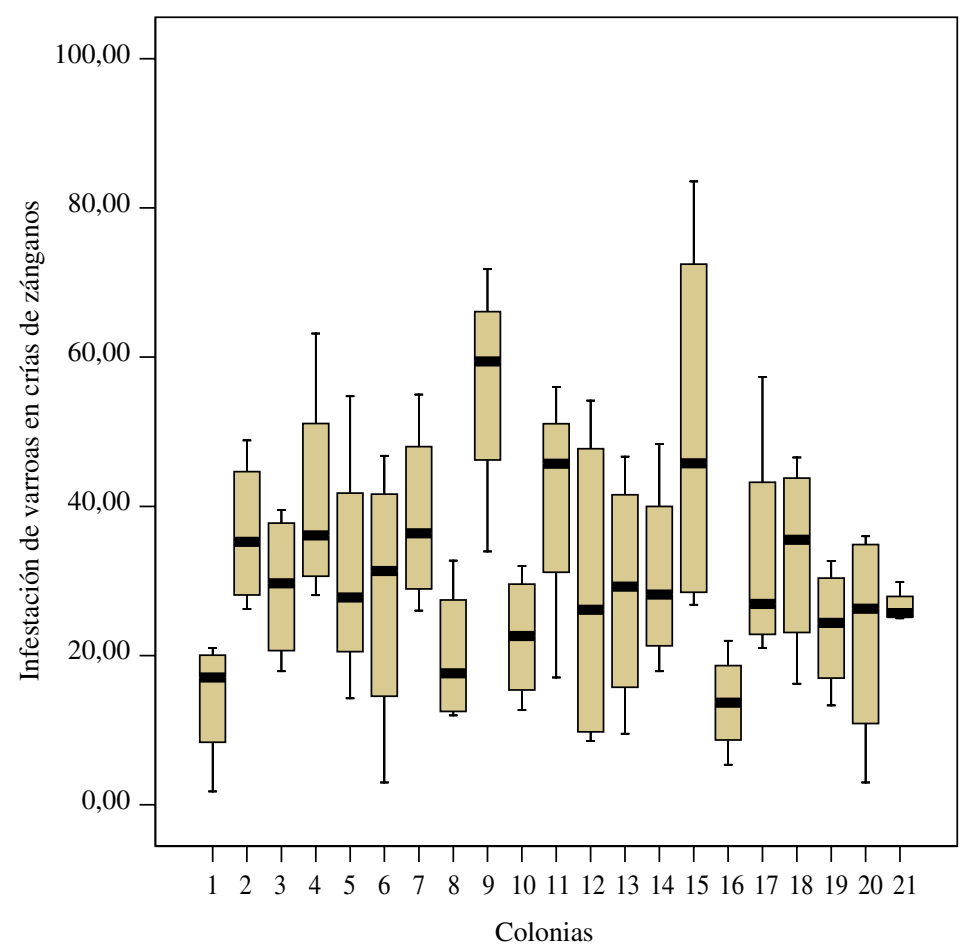

Figura 4. Porcentaje de infestación de varroas en crías de zángano por colonia. 


\section{CONCLUSIONES}

En este estudio no se logró demostrar la existencia de relación entre comportamiento higiénico de Apis mellifera e infestación de varroas, presentándose inclusive diferencias en la misma colmena en las distintas mediciones, debido a los factores externos que inciden en esta relación.

\section{LITERATURA CITADA}

ANDERE, C.; PAlaCiO, M.; DElgado, P.; FIGINI, E.; RODRÍGUEZ, E.; COLOMBANI, M. y BEDASCARRASBURE, E. 2001. Relationship Between Defensive and Hygienic Behavior in a Honeybee (Apis mellifera L.) Population. [en línea]: Proceedings of the 37th International Apicultural Congress, 28 October - 1 November, Durban, South Africa. Documento electrónico obtenido de internet. [fecha de consulta: 25 de mayo 2006]. Disponible en: http://www.inta.gov.ar/balcarce/info/documentos/alter/ apic/apismell.pdf

ARECHAVALETA, M.Y GUZMÁN-NOVOA, E. 2001. Relative Effect of Four Characteristics that Restrain the Population Growth of the Mite Varroa destructor in Honey Bee (Apis mellifera) Colonies. Apidologie (32):157-174

BENÍTEZ, R. Y MEDINA, L. 2001. Reinvasión del ácaro Varroa destructor en colonias de abejas africanizadas en Yucatán. [en linea]: IX Seminario Americano de Apicultura, Tepic, Nayarit, México 154-158. Documento electrónico obtenido de internet. 2001 [fecha de consulta: 17 de junio 2006]. Disponible en: http://www.ccba.uady.mx/cuerpos/ API/Personal/LuisMedina/Benitez_2001_SAA_ReinvasionVarroa.PDF

BOECKING O. AND DRESCHER W. 1992. The removal response of Apis mellifera $\mathrm{L}$. colonies to brood in wax and plastic cells after artificial and natural infestation with Varroa jacobsoni Oud. and to freeze-killed brood. Exp. Appl. Acarol. 16, 321-329.

CALDERONE, N.; LIN, S. Y KUENEN, L. 2002. Differential Infestation of Honey Bee, Apis mellifera, Worker and Queen Brood by the Parasitic Mite Varroa destructor. Apidologie (33): 389-398.

CHÁVEZ, E. Y GARCÍA, C. 1993. La Varroa Parásito de las Abejas [en línea]: Gobierno de Canarias, Consejería de Agricultura, Ganadería, Pesca y Alimentación, Servicio de Desarrollo Ganadero. Documento electrónico obtenido de internet. [Fecha de consulta: 15 de mayo 2006]. Disponible en: http://www.gobcan.es/agricultura/otros/publicaciones/ cuadernos/varroa.pdf.

DE LAYENS, G. Y BONNIER, G. 2001. Curso Completo de Apicultura Cuidado de un Colmenar Aislado. Editorial Omega. 411 p.

DUAY, P.; DE JONG, D. Y ENGELS, W. 2003. Weight Loss in Drone Pupae (Apis mellifera) Multiply Infested by Varroa destructor Mites. Apidologie (34): 61-65.

FLORES, J.; RUIZ, J.; PUERTA, F.; CAMPANO, F.; PADILLA, F. Y BUSTOS, M. 1998. El Grooming en Apis mellifera Iberica frente a Varroa jacobsoni Oud. Archivos de Zootecnia (47): 213-218.
Los resultados evidencian que en periodos de alta presencia de zánganos en cría, se hace necesario evaluar su infestación, siendo ésta 4 veces más alta que en cría de obrera.

Los promedios de infestación de varroa en la mayoría de las colonias se mantuvieron constantes. En abeja adulta el promedio fue $4,5 \%$ y $7 \%$ en cría de abeja obrera, encontrándose bajo el parámetro de control inmediato.

\section{GILLIAM, M.; TABER, S. AND RICHARDSON, G.V. 1983.} Hygienic behavior of honey bees in relation to chalkbrood disease. Apidologie, 14:29-39.

GRAMACHO, K. Y GONÇALVES, L. 1994. Estudo comparativo dos metodos de congelamento perfuração de crias para avalicão do comportamento higiênico em abelhas africanizadas (resumen) pag. 342 Anais do X Congresso Brasileiro de Apicultura. 14 a 18 agosto de 1994. Pousada do rio Quente-Goiás (Brasil).

GONÇALVES, L. Y GRAMACHO, K. 2000. Comportamento Higiênico de Abelhas Apis mellifera: Crias de Operárias Versus Crias de Zangões. Anais de IV Encontro Sobre Abelhas Ribeirão Preto (Brasil). 6 a 9 septiembre de 2000. pp. 66-69.

GRAMACHO, K. Y GONÇALVES, L. 2002. Melhoramento Genético de Abelhas com Base no Comportamento Higiênico. XIV Congresso Brasileiro de Apicultura Conbrapi Brasil 2002. pp. 188-190.

HARBO, J. AND HARRIS, J. 1999. Heritability in honey bees (Hymenoptera: Apidae) of characteristics associated with resistance to Varroa jacobsoni (Mesostigmata: Varroidae). Journal of Economic Entomology 92(2): 261-265.

HUERAMAN, D. 2002. Efecto de las condiciones climáticas cobre el comportamiento higiénico de abejas Apis mellifera L. en la Novena Región, Chile. Tesis (Ingeniero Agrónomo). Temuco, IX Región, Chile, Universidad Católica de Temuco, Facultad de Ciencias Agropecuaria y Forestales, Escuela de Agronomía. $82 \mathrm{p}$.

IBRAHIM, A. AND SPIVAK, M. 2006. The relationship between hygienic behavior and suppression of mite reproduction as honey bee (Apis mellifera) mechanisms of resistance to Varroa destructor. Apidologie (37): 31-40.

JONG, D; GONCALVES, L; MORSE, R 1984. Dependence of Climate on the Virulence of Varroa jacobsoni. Bee World 65: 117-121.

INVERNIZZI, C. 2001. Resistencia a la Enfermedad de Cría Yesificada por Colonias de Apis mellifera con Eficiente Comportamiento Higiénico (Hymenoptera, Apidae). Iheringia, Sér. Zool., Porto Alegre (91): 109-114, 27 de novembro de 2001.

JANMAAT, A. Y WINSTON, M. 2000. Removal of Varroa jacobsoni Infested Brood in Honey Bee Colonies With Differing Pollen Stores. Apidologie (31): 377-385.

KAMEL, S.; STRANGE, J. Y SHEPPARD, W. 2003. A Scientific Note on Hygienic Behavior in Apis mellifera lamarckii and A. m. carnica in Egypt. Apidologie (34): 189-190.

KRALJ, J. 2004. Parasite-host interactions between Varroa destructor Anderson and Trueman and Apis mellifera L.: 
Influence of parasitism on flight behaviour and on the loss of infested foragers. [en línea]: Dissertation zur Erlangung des Doktorgrades der Naturwissenschaften. Documento electrónico obtenido de internet. 2004 [fecha de consulta: 22 de mayo 2006]. Disponible en: http://www.digitalnaknjiznica.bf.uni-lj.si/dd_kralj_jasna.pdf

MEDINA, L.; MARTIN, S.; ESPINOSA, L. Y RATNIEKS, F. 2002. Reproduction of Varroa destructor in Worker Brood of Africanized Honey Bees (Apis mellifera). Experimental and Applied Acarology (27): 79-88.

MONDRAGÓN, L.; SPIVAK, M. Y VANDAME, R. 2005. A Multifactorial Study of the Resistance of Honeybees Apis mellifera to the Mite Varroa destructor Over One Year in México.Apidologie (36): 345-358.

MORETTO, G. 1996. Influência do Comportamento Higiênico no Grau de Determinado pelo Ácaro Varroa jacobsoni em Colônias de Abelhas Apis mellifera Africanizadas. V Congreso Ibero Latinoamericano de Apicultura. 30 de mayo a 02 junio 1996, Mercedes (Uruguay).

NEWTON, D. C. \& OSTASIEWSKI, N. J. 1986. A simplified bioassay for behavioral resistance to American foulbrood in honey bees (Apis mellifera L.). Am. Bee J., Hamilton, 126: 278.

OLDROYD, B. 1996. Evaluation of Australian commercial honey bees for hygienic behaviour, a critical character for tolerance to chalkbrood. Australian Journal of Experimental Agriculture. 36 (6): 625-629.

PALACIO,A.; FIGINI, E.; RODRÍGUEZ, E.; RUFFINENGO, S. 1996. Evaluación de Distintos Métodos Utilizados para Estimar el Comportamiento Higiénico en la Abeja Melífera. (resumen) V Congreso Ibero Latinoamericano de Apicultura. 30 de mayo a 02 junio 1996, Mercedes (Uruguay).

PALACIO, M.; FIGINI, E.; RUFFINENGO, S.; RODRÍGUEZ, E.; DEL HOYO, M. Y BEDASCARRASBURE, E. 2000. Changes in a population of Apis mellifera $\mathrm{L}$. selected for hygienic behaviour and its relation to brood disease tolerance. Apidologie (31): 471-478.

PALACIO, M.; FLORES, J.; FIGINI, E.; RUFFINENGO, S.; ESCANDE, A.; BEDASCARRASBURE, E.; RODRÍGUEZ, E. Y GONÇALVES, L. 2005. Evaluation of the Time of Uncapping and Removing Dead Brood from Cells by Hygienic and Non-Hygienic Honey Bees. Genetics and Molecular Research. 4 (1): 105-114.
ROBAUX, P, 1988. Varroa jacobsoni: Problems with diagnosis and control In Europa. In Nedham, G; Page R; Delfinado - Baker, M; Bowman, CE (ed) Africanizad honey bee and bee mites. Ellis Horwoord; Chichester, U.K; pp. 370-372.

SPIVAK, MY GILLIAM, 1993. Facultative expression of hygienic behavior of honey bees in relation to disease resistence. Journal of Apicultural Research. 32 (3-4): 147-157.

SPIVAK, M. AND G.S. REUTER. 1997. Successful queen rearing. Short course by University of MN Dept. of Entomology and University of MN Extension Service.

SPIVAK, M. Y DOWNEY, D. 1998 Field Assays for hygienic behaviour in Honey bees ( Hymenoptera: Apidae) Apiculture And Social Insects. 91 (1): 64-70.

SPIVAK, M. Y REUTER, G. 2001. Resistance to American foulbrood disease by honey bee colonies Apis mellifera bred for hygienic behavior. Apidologie (32): 555-565.

VANDAME, R. 2000. Control Alternativo de Varroa en Apicultura. [en línea]: Proyecto 'Abejas de Chiapas' Edición 2.2. Documento electrónico obtenido de internet. 2000 [fecha de consulta: 22 de junio 2006]. Disponible en: http://www. beekeeping.com/articulos/control_varroa/curso2.htm.

VANDAME, R.; COLIN, M. Y OTERO, G. 2000. Abejas Europeas y Abejas Africanizadas en México Tolerancia a Varroa jacobsoni. [en línea]: Tercera parte: explicación de la tolerancia. Documento electrónico obtenido de internet. 2000 [fecha de consulta: 22 de junio 2006]. Disponible en: http://www.beekeeping.com/articulos/vandame/ vandame3_sp.htm.

VICARIO, E. Y MEDINA, L. 1999. Uso del ácido fórmico y Timol en el control del ácaro Varroa jacobsoni en Yucatán, México: Resultados preliminares. [en línea]: XIII Seminario de Agricultura, Morelia, Michoacán, México 35-39. Documento electrónico obtenido de internet. 1999 [fecha de consulta: 22 de junio 2006]. Disponible en: http://www.uady. $\mathrm{mx} / \sim$ veterina/cuerpos/apicultura/apiculturaweb/luismedina/ vicario_1999_saa_varroa-timol-formico.pdf.

VIEIRA, J. Y GONÇALVES, L. 2000. Comportamento de Remoção de Ácaros de Células de Crias de Operárias de Abelhas Africanizadas. Anais de IV Encontro Sobre Abelhas Ribeirao Preto (Brasil). 6 a 9 septiembre de 2000. pp. 265-269. 